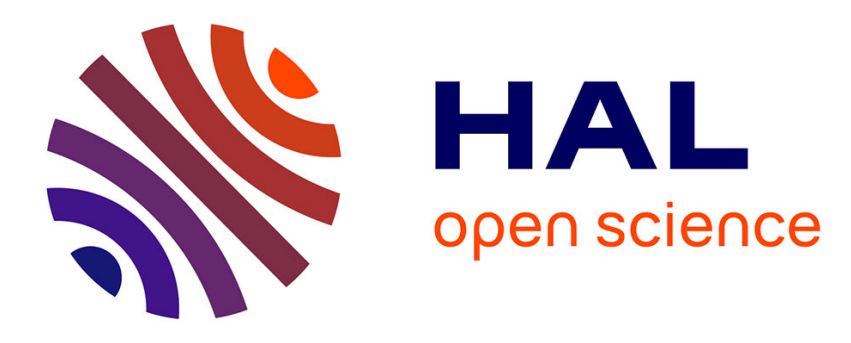

\title{
Discussion of Electronic Properties of Quasicrystals
}

\author{
Didier Mayou
}

\section{To cite this version:}

Didier Mayou. Discussion of Electronic Properties of Quasicrystals. Philosophical Magazine, 2008, 88 (13-15), pp.2209-2215. 10.1080/14786430802307660 . hal-00513931

\section{HAL Id: hal-00513931 \\ https://hal.science/hal-00513931}

Submitted on 1 Sep 2010

HAL is a multi-disciplinary open access archive for the deposit and dissemination of scientific research documents, whether they are published or not. The documents may come from teaching and research institutions in France or abroad, or from public or private research centers.
L'archive ouverte pluridisciplinaire HAL, est destinée au dépôt et à la diffusion de documents scientifiques de niveau recherche, publiés ou non, émanant des établissements d'enseignement et de recherche français ou étrangers, des laboratoires publics ou privés. 


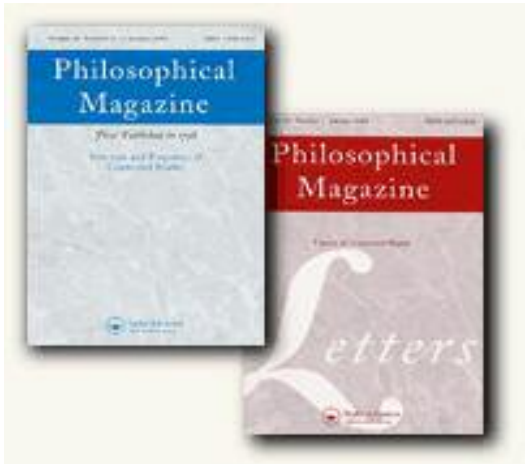

\section{Discussion of Electronic Properties of Quasicrystals}

\begin{tabular}{|c|c|}
\hline Journal: & Philosophical Magazine \& Philosophical Magazine Letters \\
\hline Manuscript ID: & TPHM-08-Jan-0009.R1 \\
\hline Journal Selection: & Philosophical Magazine \\
\hline $\begin{array}{r}\text { Date Submitted by the } \\
\text { Author: }\end{array}$ & 18-Jun-2008 \\
\hline Complete List of Authors: & Mayou, Didier; Institut Néel/CNRS, MCMF \\
\hline Keywords: & aperiodic materials, electronic properties \\
\hline Keywords (user supplied): & aperiodic materials, electronic properties \\
\hline \multicolumn{2}{|c|}{$\begin{array}{l}\text { Note: The following files were submitted by the author for peer review, but cannot be converted } \\
\text { to PDF. You must view these files (e.g. movies) online. }\end{array}$} \\
\hline Mayoufinal.tex & \\
\hline
\end{tabular}

\section{S) ScholaroNE \\ Manuscript Central}




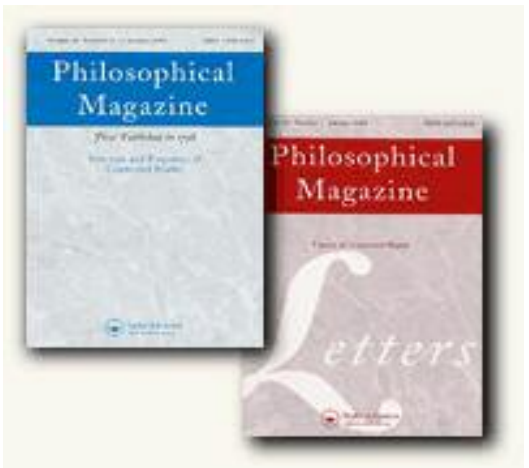

\section{Discussion of Electronic Properties of Quasicrystals}

\begin{tabular}{|r|l|}
\hline Journal: & Philosophical Magazine \& Philosophical Magazine Letters \\
\hline Manuscript ID: & draft \\
\hline Journal Selection: & Philosophical Magazine \\
\hline $\begin{array}{r}\text { Date Submitted by the } \\
\text { Author: }\end{array}$ & n/a \\
\hline Complete List of Authors: & Mayou, Didier; Institut Néel/CNRS, MCMF \\
\hline Keywords: & aperiodic materials, electronic properties \\
\hline Keywords (user supplied): & aperiodic materials, electronic properties \\
\hline & \\
\hline $\begin{array}{l}\text { Note: The following files were submitted by the author for peer review, but cannot be converted } \\
\text { to PDF. You must view these files (e.g. movies) online. }\end{array}$ \\
\hline Mayoufinal.tex
\end{tabular}

\section{ScholaronE" \\ Manuscript Central}




\title{
Discussion of Electronic Properties of Quasicrystals
}

\author{
Didier MAYOU \\ Institut Néel, CNRS and Université Joseph Fourier, Bât D, 25 av. des Martyrs, \\ B.P. 166, 38042 Grenoble Cedex 9, France \\ (June 1, 2008)
}

\begin{abstract}
This article gives a short review of some important achievements in the field of electronic properties of quasicrystals. It focuses essentially on : the nature of quasicrystals as Hume-Rothery alloys, the energetics of quasicrystals and related phases, the magnetic properties, the localization of electronic states, the transport properties. For each part one emphasizes some promising directions of research. We end by listing some problems that are related to that of electrons in a quasiperiodic potential.
\end{abstract}

\section{Introduction}

Since the discovery of quasicrystals one of the major question was that of the electronic properties induced by the new quasiperiodic order. Indeed in the case of periodic crystals the electronic states can be classified by virtue of the Bloch theorem and many fundamental properties can be deduced from this theorem. In quasiperiodic systems the equivalent of the Bloch theorem does not exist. This makes their electronic properties specific. When dealing with the electronic properties of quasicrystals it is important to keep in mind that the level of precision that is needed, in the description of the electronic structure, depends on the property of interest. For example the electronic band energy, which is of fundamental interest for stability and lattice dynamics, is a local quantity and thus one expect to get a reasonnable descrition of the energetics just by considering a local approach in real space or equivalently by considering details of the electronic structure of the order of a fraction of an $\mathrm{eV}$. The existence of local magnetic moments (for example on $\mathrm{Mn}$ in $\mathrm{Al}$ based quasicrystals) should be described also by considering local environment effects. On the contrary transport properties are expected to be sensitive to the atomic order on a larger scale than energetic quantities, and require a finer description of the electronic structure.

\section{Quasicrystals as Hume-Rothery alloys}

Very early Bancel et al. [?] and Friedel et al. [?] insisted on the fact that quasicrystalline phases where Hume-Rothery alloys, also named electron compounds. According to Hume-Rothery the stability of these alloys results from a nesting condition between the Fermi surface and strong Fourier components $G$ of the potentiel felt by valence electrons. One has $G \simeq 2 k_{F}$ where $k_{F}$ is the Fermi wavevector. The HumeRothery concept has been a guiding principle for discovering numerous new quasicrystalline phases [?]. A direct consequence of the Hume-Rothery principle is the existence of a pseudo-gap in the electronic density close to the Fermi energy. The pseudogap in these phases is confirmed by NMR experiments [?], X-ray measurements and photoemission [?,?]. For icosahedral phases containing transition metal (TM) elements, specific heat measurement indicate a DOS at $E_{\mathrm{F}}$ of the order of $1 / 3$ of the free electron value for i- $\mathrm{AlCuFe}$ and 1/10 for i-AlCuRu and i-AlPdRe [?,?]. The role of the transition metal (TM, TM = Ti, V, Cr, Mn, Fe, $\mathrm{Co}, \mathrm{Ni}$ ) element in the pseudogap formation has been shown from ab-initio calculations [?,?] and results from a strong sp-d coupling associated to an ordered sub-lattice of TM atoms. It was also shown that, as a result of the sp-d hybridization, transition metal (TM) elements play an important role on the unusual

*Corresponding author. Email: didier.mayou@grenoble.cnrs.fr

Philosophical Magazine

ISSN 1478-6435 print/ISSN 1478-6443 online (c) 200x Taylor \& Francis

http://www.tandf.co.uk/journals

DOI: $10.1080 / 1478643 Y Y \operatorname{xxxxxxxx}$ 
transport properties of quasicrystals and related phases [?,?,?,?,?]. The negative valency of transition metal elements, introduced by Raynor, is fundamental in studying the stability of quasicrystals and in predicting new stable phases [?,?]. As shown in [?] the negative valency is a consequence of the effect of sp-d hybridization on the density of states below the pseudo-gap. Quite generally the electronic structure and the atomic structure of Quasicrystals are related in a delicate way and a new exciting field of study is the case of surfaces [?].

\section{$3 \quad$ From oscillating atomic pair potentials to stability and lattice dynamics}

The energetics of Hume-Rothery alloys can also be described in simple terms. Indeed the atoms can be viewed as scatterers for the free valence electrons so that the energy of a phase is the sum of a term that depends only on the electronic density of the alloy plus a sum of effective interactions between all pairs of atoms. The pairs interaction thus determine the atomic structure for a given density.

Recently many progresses have been done by using a proper description of the energetics of these alloys. For example it has been possible to justify the Matching Rules in some models of binary decagonal quasicrystal closely related to actual aluminum transition metal structures [?]. The stability of twodimensional monoatomic model systems has also been studied [?] using effective pair potential that presents oscillations in real space just as in Hume-Rothery alloys (Friedel oscillations). The variation of energy when the atoms move around their equilibrium position gives also access to the energetics of phonons and to the lattice dynamics. This allows to include the role of phonons in the thermodynamics potentials of high temperature phases and gives a fine understanding of the stability of some decagonal Al-Co-Ni structures [?]. The good description of phonons dispersion relations obtained by this approach has been experimentally confirmed [?] . Progresses in this direction will proceed and should greatly improve our understanding of the stability of quasicrystals and of their phonon properties .

\section{Magnetism}

Magnetism in quasicrystals is a fascinating field of investigation, obviously in the case of R-Mg-Zn QCs with $\mathrm{R}$ a rare earth which carries a magnetic moment, but also in the case of Al-based QC containing $\mathrm{Mn}$, which generated puzzling questions since the very beginning of the QC story. The fact that most of the $\mathrm{Mn}$ atoms are non magnetic in Al-Mn and Al-Pd-Mn QCs and approximants can be understood from their particular electronic structure [?]. Yet it is still difficult to determine where all the magnetic atoms are and what exactly, in their local environment, induces magnetic moments [?].

A remarquable result found by Hippert and Préjean [?] is that AlPdMn QCs turn out to be a model system for studying experimentally the Kondo vs RKKY competition in the absence of long range magnetic ordering, which is a fundamental problem in magnetism. Indeed in AlPdMn the magnetic Mn atoms undergo standard magnetic interactions, just like in any dilute magnetic alloy. These interactions are the Kondo coupling between a single moment and the conduction electron spins and RKKY interactions between moments leading to a spin glass transition. However, because the characteristic energy scales of these interactions lie in the Kelvin range, it becomes possible to study the Kondo vs RKKY competition whereas it is not possible in standard dilute magnetic alloys like CuMn.

Another remarquable result due to Préjean and coworkers [?,?] is that in AlPdMn quasicrystals magnetic properties, transport properties and structural quality are intimately linked. The signature is that the conductivity, as opposed to the resistivity for normal metals containing magnetic Kondo impurity , exhibits a Kondo-type $\ln \mathrm{T}$ increase at decreasing temperature. This striking effect is a manifestation of the unconventional transport properties of quasicrystals.

In the case of R-Mg-Zn QCs each rare earth atom (R) carries a magnetic moment. One of the most interesting question in the magnetism of these quasicrystals is that of the long range magnetic order and the possibility of a new kind of spin glass. No long range magnetic order has been observed yet and the dynamics of spin correlations requires more studies [?,?]. 


\section{Electron localisation by atomic clusters and by long range quasiperiodic potential}

One of the characteristics of the quasicrystals and their approximants, is the occurrence of atomic clusters on a scale of 10-30 $\AA$ [?]. These clusters are not perfectly well defined because some of them overlap, and in addition there are the so-called glue atoms. Yet the role of clusters has been much debated after the work of by C. Janot et al. [?]. As shown in [?], the DOS due to a Transition Metal cluster exhibits peaks. The width $\delta E$ of the most narrow peaks $(\delta E \simeq 10-100 \mathrm{meV})$ is comparable to the fine peaks of the calculated DOS in the approximants. Each peak indicates a resonance due to the scattering by the cluster. and correspond to states which are nearly localized by the cluster. Some recent experiments with break junction show some fine structure in the DOS that could disappear when averaging the DOS on large spatial scale [?]. A clear experimental characterization of a spiky DOS is still lacking and the condition for its observation is also debatted.

The long range quasiperiodic potential tends also to localize states. In $1 \mathrm{D}$ the eigenstates are neither extended like in crystals nor exponentially localized like in disordered insulators but are "critical" which is an algebraic localization. Most studies of electronic localization have focused on 1D systems for which one can use many powerful tool. There are evidence that in in $2 \mathrm{D}$ and $3 \mathrm{D}$ systems the eigenstates are also critical but there is a lack of studies in these systems [?]. Exact diagonalization is rapidly limited in $2 \mathrm{D}$ and $3 \mathrm{D}$ yet there are efficient tools to study quantum diffusion or return probability on systems containing up to several $10^{6}$ orbitals that characterize well the nature of states [?]. One challenge is to find quasiperiodic models with diffusion coefficients that are less than $1 / 2$. Indeed such models should have transport properties very similar to those experimenatlly observed [?] (see below).

\section{Unconventional electronic transport in quasicrystals}

The first quasiperiodic alloys AlMn were metastable and contained many structural defects. As a consequence they had conduction properties similar to those of amorphous metals with resistivities in the range $100-500 \mu \Omega \mathrm{cm}[?]$. The discovery of the stable AlCuFe icosahedral phase, having a high structural order and very high resistivity, of the order of $10000 \mu \Omega \mathrm{cm}$, gave a considerable interest in their conduction properties. The conductivity presented a set of characteristics that was neither that of semi-conductors nor that of normal metals [?,?,?]. The density of states in $\mathrm{AlCuFe}$ is smaller than in $\mathrm{Al}$, about one third of that of pure $\mathrm{Al}$, but still largely metallic. In addition weak-localization effects are observed that are typical of amorphous metals. Besides, the resistivity of some quasicrystals and approximants (AlPdMn, $\mathrm{AlCuFe}$ ) decreases when temperature increases and after annealing a sample, with a strong reduction of its structural defects, its resistivity increases. These behaviors are characteristics of semiconductors. Yet some facts are unique to these quasicrystals and unobserved in metals and semiconductors. Indeed their conductivity follows approximatively the so-called "inverse Mathiessen rule" [?,?] and is the sum of a term that depends only on the sample plus a term that depends only on the temperature $\sigma(T)=\sigma_{0}+\Delta \sigma(T)$. In addition the Drude peak is absent and the optical conductivity presents a linear frequency dependence $[?, ?]$. Experiments on quasicrystals and approximants with various size of the unit cell show that the local atomic order on the length scale of the unit cell, i.e. $10-30 \AA$, determines their transport properties [?].

In 1993 another breakthrough was the discovery of AlPdRe which had resistivities in the range of $10^{6} \mu \Omega \mathrm{cm}[?, ?, ?, ?, ?, ?, ?]$, although the DOS still has a metallic character. This material displays a strong decrease of the conductivity when the temperature is reduced and the conductivity value can fall below $1(\Omega \mathrm{cm})^{-1}$ at $4 \mathrm{~K}$. Although the behavior depends strongly on the composition and the preparation of the sample, many authors $[?, ?, ?, ?, ?, ?, ?]$ reported that AlPdRe quasicrystal is very close to the metalinsulator transition. Three successive regimes are revealed [?] as the temperature is increased to room temperature: a low temperature variable range hopping-like behavior, followed by a Thouless regime and a high temperature critical regime. The transport properties of AlPdRe are difficult to study experimentally in particular because of the difficulty to get good and homogeneous samples. Yet it is still a challenge to get the best experimental description of their conduction properties. 


\section{Mechanism of electronic transport : breakdown of the semi-classical model}

The standard theory of electronic transport in metals is the semi-classical Bloch-Boltzmann theory. According to that theory the electrons are wave-packets that propagates ballistically and are scattered by defects which change their direction of propagation leading to a long time diffusive motion. In quasicrystals and approximants this picture is no more valid [?,?]. Indeed in quasicrystals the propagation between scattering events in the perfect structure is not ballistic but follows a power law $\Delta X^{2}(E, t) \propto t^{2 \beta} . \Delta X^{2}(E, t)$ is the spreading of states with energy $E$ after a time $t$ in a given direction $X$ and the positive diffusion exponent $\beta \leq 1$. This changes the dependence of the conductivity on scattering time and frequency as shown by the Generalized Drude formula [?]. In particular this formula predicts that if $\beta<1 / 2$ the conduction properties are unconventional and in agreement with those observed experimentally. In approximants the propagation of electrons between scattering events depends on two channels the standard one (ballistic) and another one which describes the propagation of electrons in one unit cell. So $\Delta X^{2}(E, t)=V_{\mathrm{B}}(E)^{2} t^{2}+\Delta X_{\mathrm{NB}}^{2}(E, t)$, where $V_{\mathrm{B}}$ is the band velocity at energy $E$ and $\Delta X_{\mathrm{NB}}(E, t)$ is bounded by $L / 2$ where $L$ is the length of the unit cell in the $X$ direction. The link between quantum diffusion in approximants and in quasicrystals is discussed in [?]. In standard metallic crystals, at the scale of realistic scattering times, $\Delta X_{\mathrm{NB}}^{2}(t)$ term is negligible with respect to the Boltzmann term $V_{\mathrm{B}}(E)^{2} t^{2}$ and the semi-classical theory is applicable. Yet for approximants, both terms $V_{\mathrm{B}}(E)^{2} t^{2}$ and $\Delta X_{\mathrm{NB}}^{2}$ can have the same magnitude around a typical scattering time value $\sim 10^{-14} s$ [?] so that it is possible to observe cases where one or the other term dominates. If the non Boltzmann term dominates then unconventional transport properties are predicted in agreement with experimental results [?]. The optical conductivity $\sigma(\omega)$ can also be calculated as the sum of two terms [?]. The absence of Drude peak is explained if the Boltzmann term is negligible. The metal-insulator transition (Anderson transition) is also deeply affected by the non ballistic propagation [?].

\section{Electrons in a quasiperiodic potential and related problems}

The problem of electrons in a quasiperiodic potential is related to other very interesting physical problems.

The first and obvious one is that of phonons in quasicrystals. Indeed phonons are also wavelike excitations and share fundamental properties with electrons. In particular the heat transport by phonons presents deep analogies with current transport by electrons [?]. It will be interesting in the next years to developp this analogy further, for example for the analysis of quantum diffusion of phonons (heat diffusion).

Also flat bands, which are at the heart of the transport properties of quasicrystals, could be observed in other electronic systems [?]. For example flat bands exist in polaronic systems (strong electron-lattice coupling [?]) and also in correlated systems, like heavy Fermions. Indeed in these systems transport properties present analogies with those of quasicrystals [?]. Flat bands can also be artificially produced in nanostructures : for example by applying a strong magnetic fields to electrons in a large ribbon.

As a last example one may consider photonic and phononic crystals. Many remarkable properties of these systems arise when a gap in the excitation spectrum is produced by the periodic, or quasiperiodic, arrangement of the artificial structure. The higher rotationnal symmetry of quasiperiodic systems favours omnidirectionnal band gaps and is thus efficient to produce artificial structures with useful properties [?]. However quasiperiodic photonic or phononic crystals open new possibilities and new questions and in particular with the role of the gap and the existence of in-gap states, or new possibilities to exploit non linear effects [?].

\section{Acknowledgements}

I am grateful to many colleagues : Pr N. W. Ashcroft, Pr J. Bellissard, C. Berger, F. Cyrot-Lackmann, J. Delahaye, Pr T. Fujiwara, Pr J. Friedel, T. Grenet, Pr F. Hippert, J.P. Julien, Pr T. Klein, L. Magaud, J. J. Préjean, P. Quémerais, S. Roche, F. Triozon. I express special thanks to G. Trambly de Laissardière . This article is motivated by a discussion on the electronic properties of quasicrystals that took place during the conference "Quasicrystals - The Silver Jubilee" and was initiated by the Conference Chair, Ron 
Lifshitz. The discussion was led by the author who was assisted by a panel of experts consisting of Janez Dolinsek, Francoise Hippert, and Guy Trambly de Laissardiere, to whom the author is grateful. It should be noted that the current article presents the author's personal thoughts and views on the current state of affairs, and is not an attempt to provide an accurate record of the panel discussion.

\section{References}

[1] Bancel PA, Heiney PA Phys.Rev. B 337917 (1986)

[2] J. Friedel, Denoyer F. C.R. Acad.Sci.Paris Ser II, 305171 (1987).

[3] A.P. Tsaï this issue

[4] F. Hippert, L. Kandel, Y. Calvayrac, and B. Dubost, Phys. Rev. Lett. 692086 (1992).

[5] E. Belin and Z. Danhkazi, J. Non-Cryst. Solids. 153-154 298 (1993).

[6] E. Belin-Ferré, J. Phys.: Condens. Matter 14 R789 (2002); E. Belin-Ferré, in this issue.

[7] S. J. Poon, Adv. Phys. 41303 (1992).

[8] C. Berger, in Lectures on Quasicrystals. edited by F. Hippert, D. Gratias (Les Editions de Physique, Les Ulis 1994) pp. 463.

[9] G. Trambly de Laissardière, D. Nguyen Manh, L. Magaud, J. P. Julien, F. Cyrot-Lackmann and D. Mayou, Phys. Rev. B 527920 (1995).

[10] G. Trambly de Laissardière, D. Nguyen Manh and D. Mayou, Prog. Mater. Sci. 50679 (2005).

[11] E. Belin and D. Mayou, Phys. Scr. T 49356 (1993).

[12] C. Berger, E. Belin and D. Mayou, Ann. Chim. Mater (Paris) 18485 (1993).

[13] D. Mayou, F. Cyrot-Lackmann, G. Trambly de Laissardière and T. Klein, J. Non-Cryst. Solids 153-154412 (1993).

[14] Z. Dankházi, G. Trambly de Laissardière, D. Nguyen-Manh, E. Belin and Mayou, J. Phys.: Condens. Matter 53339 (1993).

[15] G. Trambly de Laissardière, Z. Dankházi, E. Belin, A. Sadoc, D. Nguyen-Manh, D. Mayou, M. A. Keegan and D. Papaconstantopoulos, Phys. Rev B 5114035 (1995).

[16] Y. Ishii et al this issue

[17] M Krajci et al this issue

[18] C. Henley et al this issue

[19] M. Engel and H.R. Trebin this issue

[20] M. Widom et al this issue

[21] M. de Boissieu et al this issue

[22] G. Trambly de Laissardière and D. Mayou, Phys. Rev. Lett. 853273 (2000).

[23] Godonyuk, Vekilov, and Isaev, this issue (Local environment and magnetism in icosahedral quasicrystals)

[24] F. Hippert and J.J. Préjean this issue

[25] R. Lifshitz. Phys. Rev. Lett. 80 (1998) 2717-2720. (Symmetry of magnetically ordered quasicrystals), R. Lifshitz. Materials Science and Engineering A 294 (2000) 508-511. (Magnetic quasicrystals: What can we expect to see in their neutron diffraction data?), R. Lifshitz and S. Even-Dar Mandel. Acta Cryst. A60 (2004) 167-178. (Magnetically-ordered quasicrystals: Enumeration of spin groups and calculation of magnetic selection rules)

[26] J. J. Préjean, C. Berger, A .Sulpice and Y. Calvayrac, Phys. Rev. B 65 R140203 (2002).

[27] J.J. Préjean, E. Lhtel, A. Sulpice, F. Hippert Phys. Rev. B 73214205 (2006).

[28] D. Gratias, F. Puyraimond, M. Quiquandon and A. Katz, Phys. Rev. B 6324202 (2000).

[29] C. Janot and M. de Boissieu, Phys. Rev. Lett. 721674 (1994).

[30] G. Trambly de Laissardière and D. Mayou, Phys. Rev. B 552890 (1997).G. Trambly de Laissardière and D. Mayou, Phys. Rev. Lett. 853273 (2000), E. Belin and D. Mayou, Phys. Scr. T 49356 (1993).

[31] Okada J.T. et al J. Phys. Soc. Japan 76033707 (2007), Widmer et al Phil. Mag. 86781 (2006)

[32] Even-Dar Mandel and Lifshitz, this issue (Electronic Energy Spectra of Square and Cubic Fibonacci Quasicrystals), Even-Dar Mandel and Lifshitz. Phil. Mag. 86 (2006) 759-764. (Electronic energy spectra and wave functions on the square Fibonacci tiling)

[33] F. Triozon, J. Vidal, R. Mosseri and D. Mayou, Phys. Rev. B 65220202 (2002).

[34] D. Mayou, G. Trambly de Laissardière, in Physics of Quasicrystals, series "Handbook of Metal Physics", edited by T. Fujiwara and Y. Ishii (Elsevier Science, Amsterdam, 2008), p. 209; arXiv:cond-mat/0701639.

[35] Trambly de Laissardiere et al, this issue (Electronic transport in AlMn(Si) and AlCuFe quasicrystals: Break-down of the semiclassical model)

[36] T. Grenet, in Quasicrystals: Current Topics, edited by E. Belin-Ferré, C. Berger, M. Quiquandon and A. Sadoc, (World Scientific, Singapore, 2000) pp. 455.

[37] D. Mayou, C. Berger, F. Cyrot-Lackmann, T. Klein and P. Lanco, Phys. Rev. Lett 703915 (1993).

[38] C. C. Homes, T. Timusk, X. Wu, Z. Altounian, A. Sahnoune and J. O. Ström-Olsen, Phys. Rev. Lett. 672694 (1991).

[39] S. E. Burkov, T. Timusk and N. W. Ashcroft, J. Phys.: Condens. Matter 49447 (1992).

[40] Dolinsek et al., this issue (Anisotropic electrical, magnetic and thermal transport properties of the Al80Cr15Fe5 decagonal approximant)

[41] P. S. Pierce, S. J. Poon and Q. Guo, Science 261737 (1993); F. S. Pierce, S. J. Poon SJ and B. D. Biggs, Phys. Rev. Lett 703919 (1993).

[42] C. Berger, T. Grenet, P. Lindqvist, P. Lanco, J. G. Grieco, G. Fourcaudot and F. Cyrot-Lackmann, Solid State Commun 87977 (1993).

[43] H. Akiyama, Y. Honda, T. Hashimoto, K. Edagawa and S. Takeuchi, Jpn. J. Appl. Phys. 32 L1003 (1993).

[44] J. Delahaye, J. P. Frison and C. Berger, Phys. Rev. Lett 814204 (1999).

[45] J. Delahaye and C. Berger, Phys. Rev. B 64094203 (2001).

[46] J. Delahaye, C. Berger and G. Fourcaudot, J. Phys.: Condens. Matter 158753 (2003).

[47] R. Rosenbaum, T. Murphy, B. Brandt, C. R. Wang, Y. L. Zhong, S. W. Wu, S. T. Lin and J. J. Lin, J. Phys.: Condens. Matter 16 821 (2004); R. Rosenbaum, B. Grushko and B. Przepiorzynski, J. of Low Temp. phys. 142101 (2006).

[48] D. Mayou, Phys. Rev. Lett 851290 (2000).

[49] G. Trambly de Laissardière, J. P. Julien and D. Mayou, Phys. Rev. Lett. 97026601 (2006).

[50] D. Mayou D. in Quasicrystals: Current Topics, edited by E. Belin-Ferré, C. Berger, M. Quiquandon and A. Sadoc, (World Scientific, Singapor, 2000) pp. 412. 
[51] P. Quémerais and S. Fratini, Mod. Phys. Lett. B 111303 (1997); Physica C 341229 (2000); S. Fratini and P. Quémerais Eur. Phys. J. B 2941 (2002).

[52] W. Steurer and D. Sutter-Widmer, J. Phys. D: Appl. Phys. 40 (2007) R229-R247. (Photonic and phononic quasicrystals)

[53] Bahabad et al., this issue, R. Lifshitz, A. Arie, and A. Bahabad. Phys. Rev. Lett. 95 (2005) 133901. (Photonic quasicrystals for nonlinear optical frequency conversion), B. Freedman et al., Nature 440 (2006) 1166. (Observation of wave and defect dynamics in nonlinear photonic quasicrystals) 